\title{
Prospective Classification over Various Handwritten Character Recognition Algorithms - A Survey
}

\author{
Pradheeba $\mathrm{U}^{\mathrm{a}, 1}$, Bhavani $\mathrm{M}^{\mathrm{b}}$ Yuvaraj $\mathrm{B} \mathrm{R}^{\mathrm{c}}$ and Krithika $\mathrm{V}^{\mathrm{d}}$ \\ ${ }^{a}$ Department of CSE, R.M.K. College of Engineering and Technology, India \\ ${ }^{\mathrm{b}}$ Department of CSE, Rajalakshmi Engineering College, India \\ ${ }^{\mathrm{c}}$ Department of IST, Anna University, India \\ ${ }^{\mathrm{d}}$ Department of R\&D and RDAF, National Institute of Wind Energy, India
}

\begin{abstract}
Handwritten Character Recognition is comprehensively used to scrutinize and appreciate characters from pictures and secret them to cutting edge substance. Character recognition is head, yet for the most part testing in the field of model recognition with a colossal number of significant applications. It has been a genuine field of investigation since the start of programming since it being a characteristic strategy for associations among PCs and individuals. Even more accurately Character recognition is the route toward recognizing and seeing characters from the data picture and converts it into ASCII or another similar machine editable design.
\end{abstract}

Keywords. Handwritten Character Recognition, OCR, PTR, CNN, Perceptron.

\section{Introduction}

Understanding the characters written manually or created reports is simple for humans as we can learn. A comparative upper limit can be whipped up to the machines in that manner by the usage of ML or DL in AI. The field which focuses with this issue is called as OCR. It is a place to study among various fields, for example seeing of various model, like manner image vision and furthermore AI. This is the area for changing the electronically and picturing text into high level character which need to be deduced by the machines [1]. By the time, we use for entering the data and the same way additional space is required by the files can be momentously diminished by the use of OCR or towards the day end, it will be demised rapidly. With the help of OCR in the financial field, actual situations where we need to handle social documents and for many critical and time sensitive documents will be handled very fast without human intervention. Optical Character Recognition will be triggered by substance and file getting and this will be happening as two distinct manners. Moreover, on the positive side that we contemplate the substance kind, OCR is of two sorts, HCR - astute recognition of handwritten substance

\footnotetext{
${ }^{1}$ Pradheeba U, Department of CSE, R.M.K. College of Engineering and Technology, India.

E-mail: prathiba.ulaganathan@gmail.com
} 
and PCR. All we need a high identifying limit on the chances of changing handwriting of individuals. Many on various occasions, the similar individuals had different making style from period to period. Then as far as OCR is concerned, the two designs which are characterized are Offline and Online recognition which could reliant upon getting the details of the reports. Detached System figures out how to see the pre-made report acquired through various data strategies. However, in the Online seeing system, the creating is seen the subsequent it is composed. The device used for the online structure is an Electric pen where it is used for creating the letters or words on the device called a digitizer and dependent on the pen improvement the data is recorded. This investigation gives an extensive connection between's different machine learning and significant learning estimations with the ultimate objective of handwritten character recognition while using the Support Vector Machine, Multilayer Perceptron, and Convolutional Neural Network for a comparative explanation The relationship between's these computations is done dependent on their exactness, bungles, and testing-getting ready time supported by plots and charts that have been assembled using matplotlib for portrayal. Hence this paper figures out how to give an assessment of different computations subject to their accuracy so the most definite estimation with insignificant chances of bumbles can be used in various usages of handwritten character recognition.

This paper gives a reasonable understanding of machine learning and significant learning computations like SVM, CNN, and MLP for handwritten character recognition. It other than gives you the information about which computation is successful in playing out the errand of character recognition. In extra spaces of this paper will inspect the associated work that has been done in this field followed by the technique and execution of the huge number of three estimations for a more charming understanding of them. At that point, it presents the end and result supported by various diagrams. Moreover, it will similarly give information about some potential future upgrades that ought to be conceivable in this field. Figure 1 underneath is a summed up block outline that portrays the overall work process of all Handwritten Recognition Algorithms.

\section{Literature Survey}

Many Algorithms have evolved according to the type of written data. These algorithms have been studied for their pros and cons for analyzing them and the core points about those are given below. Proper analysis in the world of text recognition is one of the favorite from Grimsdale in 1959 and it has become the main purpose for many researchers to start evolve many handwritten character recognition. During 1960's to 1965's the main examinations proved that use of appraisal was proposed by Eden in 1969. He proved that handwritten character are subjected to few schematic and semantic highlights and his theory was widely utilized as a work of basics of all traditional methods for approaching in space of character identification [1].

Gaurav and Bhatia [2], have suggested various pre-processing machines for being recognizable with the identification of the characters. This approach went off from different source to source in the form of picture-based report to colored and kept for change including the base one. Various systems of pre-processing and standard metrics like separate chaining, slant fix and different other methods are stated.

Salvador España-Boqueria [3], the authors have suggested that the genetic breed or half-concealed HMM to observe the handwritten substance in different modes. The 


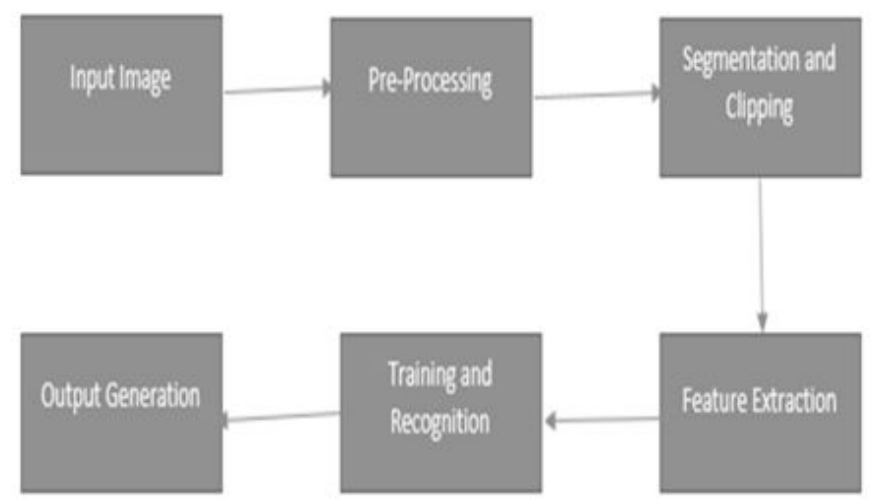

Figure 1. General Block Diagram for Recognition Algorithms

optical model's most important part was to set up with Markov and multilayer perceptron was then used to measure the possibilities.

In [4], to watch the emotionally detached handwritten letters of six noticeable Indian dialects, a modified quadratic classifier is used. A new comparable paper moreover finds out where to see the English letter and how to see them all together. For all of these, a multilayer perceptron was there and two entities were used for segment extraction such as boundary following and fourier descriptors. By checking the shape and look at their features with a glance, those characters were brought to knowledge.

Bajaj et al. [5], were on the other hand collecting artifacts about Devanangari numerals with their features like clear part, moment features, thickness are taken into consideration. To set the identification limit, this one has shared a multi classifier with purposeful quality for handwritten Devanangari numerals. Sandhya Arora in [6] clearly provided as four important features like shadow, crossing point, histogram of chain and level line fitting features being shown as metrics. In these, the shadow was projected as all around for picture character, the rest three were prepared by sharing the character picture into few mentioned segments.

Nafiz et al. [7] suggested the strategy in which it is less demanding to hold an essential removal from the pre handling stage and thereby reducing the deficiency of basic and needed information. The best of all proposed was the estimation of cable division. Same way, various methods were always giving their continuous support to this calculation with the help of maxima and minima of neighborhood. When we use the onedimensional depiction of a character which has two dimensions it immensely starts to build the energy wave of HMM for shape seeing.

In [8], a proposal was given to see the unique Tamil written character by stroke collections. The arrangement of strokes or shape based expression is meant for addressing a line of shape features. Using this approach, one can differentiate the non identified strokes and the dataset of others by string arrangement techniques in a model which is highly adaptable. Using this, every individual character was identified by recognizing all of the strokes [9]. 


\section{Analysis}

The comparison of the algorithms (Support vector machines, Multi-layered perceptron \& Convolutional neural network) is based on the characteristic chart of each algorithm on common grounds like dataset, complexity of the algorithm, accuracy of each algorithm, specification of the device used to execute the program and runtime of the algorithm, under ideal condition. The common classification over HCR algorithms are given below in Figure 2.

\subsection{Support Vector Machine}

Support Vector Machine (SVM) is a managed machine learning calculation that can be utilized in high-dimensional spaces. Character recognition utilizing SVM is generally memory-effective and additionally time-proficient [10]. In SVM, the information things are plotted in $\mathrm{n}$-dimensional space where $\mathrm{n}$ is the quantity of highlights, and a specific arrange addresses the worth of an element, grouping is performed by finding the hyperplane which is utilized to recognize the two classes. In the wake of plotting, the hyperplane which is utilized to recognize the classes is distinguished. There are primarily two kinds of SVMs, linear and non-linear SVM. The SVM in scikit-learn can support both thick and meager example vectors as info. LinearSVC is utilized in this paper for the order of MNIST datasets that utilize a Linear portion carried out with the assistance of LIBLINEAR. Different sci-unit learn libraries like NumPy, matplotlib, pandas, Sklearn and seaborn have been utilized in the execution. First and foremost, MNIST datasets are download, trailed by stacking them and perusing those CSV documents utilizing pandas. After this, a few examples are plotted and changed over to a grid followed by standardization and scaling of highlights. At long last, make a linear SVM model and disarray network is utilized to gauge the precision of the model.

\subsection{Multilayer Perception}

These limit cases are called support vectors, and thusly, the calculation is named a Support Vector Machine. A multilayer perceptron (MLP) is a class of feedforward artificial neural networks (ANN). It is isolated into three layers: input layer, covered up layer and yield layer. Each layer is characterized as an assortment of a few hubs that are alluded to as neurons and every hub is interconnected to each and every hub in the following layer. In fundamental MLP there are 3 layers yet the quantity of covered up layers can increment to any number according to the issue with no limitation on the quantity of hubs. The quantity of hubs in the information and yield layer fluctuates dependent on the quantity of qualities and obvious classes that are in the dataset. The specific number of covered up layers or number of hubs in the secret layer is hard to decide because of this current model's whimsical nature and accordingly must be chosen tentatively. Each secret layer of the model can have an alternate enactment work that is utilized for preparing. For learning purposes, a directed learning strategy called backpropagation is utilized. In the MLP, the idea of weight is utilized in the association of the hubs which gets acclimated to synchronize with every association in the preparation cycle of the model.[10]. The execution of Handwritten Character Recognition by Multilayer perceptron, otherwise called feedforward artificial neural network, is finished with the assistance of Keras 


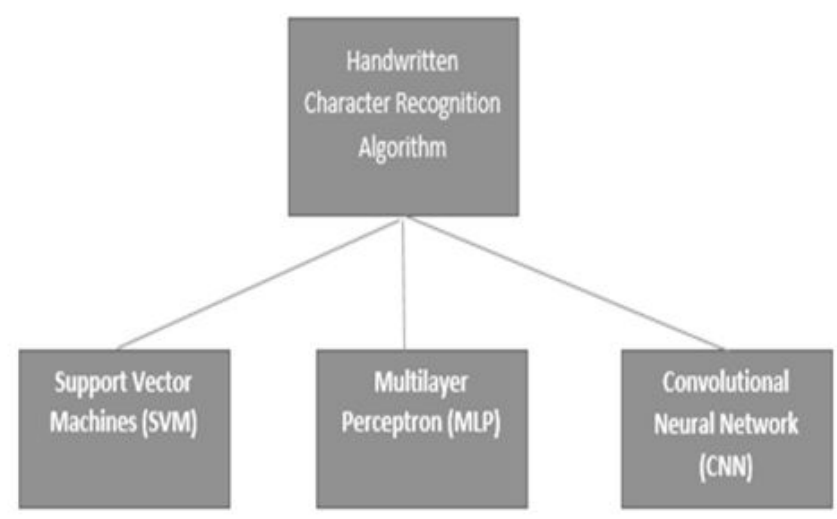

Figure 2. Classification of HCR Algorithms

module to make a MLP model of Sequential class and add individual secret layers with various actuation capacity to take a picture of $28 \times 28$ pixel size as information. When preparing and test information are free, one can follow these means to prepare a neural network in Keras. This paper utilizes a neural network with 4 secret layers and a yield layer with 62 units . The quantity of units in the mysterious layers is kept to be 512 . The examination among the talked about algorithms is classified beneath in Table 1. In the Sequential model, anybody can simply pile up layers by adding the ideal layer individually. This paper utilized the Dense layer, moreover called a completely associated layer since it is building a feedforward network in which every one of the neurons from one layer are associated with the neurons in the past layer. Aside from the Dense layer, the ReLU initiation work is added, which is needed to acquaint non-linearity with the model. Through this, non-linear choice limits are learned by the network. As it is a multiclass characterization issue, the last layer is a softmax [11] layer.

\subsection{Convolutional Neural Network}

Convolutional Neural Network is a profound learning calculation that is generally utilized for picture recognition and arrangement. It is a class of profound neural networks that require least pre-processing [12]. In $\mathrm{CNN}$, the picture is contribution to the type of little lumps instead of contributing a solitary pixel at a time, so the network can recognize dubious examples (edges) in the picture all the more proficiently. CNN comprises principally of 3 layers, an info layer, a yield layer, and numerous secret layers which incorporate Convolutional layers, pooling layers (Max and Average pooling), completely associated layers (FC), and standardization layers [13]. CNN utilizes a channel (piece) which is a variety of loads that are utilized to extricate highlights from the info picture. CNN utilizes distinctive activation capacities at each layer to add some non-linearity [14]. Further, it tends to be seen that the stature and width decline while the quantity of channels increments. At long last, the created segment lattice is utilized to foresee the output [15]. The execution of handwritten character recognition by Convolutional Neural Networks done by utilizing Keras. Utilizing Keras, a model is made layer by layer by utilizing sequential classes. The component of the info picture is set to 28(Height), 
28(Width), 1(Number of channels). After this, the model is made with the Convolutional layer as the primary layer. This layer utilizes a framework to convolve around the info information across its stature and width and concentrate highlights from it. This network is known as a Filter or Kernel. In this layer, the Depth (number of channels) of the yield picture is equivalent to the quantity of channels utilized. To build the non-linearity, an activation work that is ReLU [16] is utilized. After this, another convolutional layer is utilized in which 64 channels of similar measurements $(3,3)$ with a step of 1 and the Relu work is applied.

Table 1.: Analysis of various Handwritten Recognition Algorithms

\begin{tabular}{|c|c|c|c|c|c|}
\hline \begin{tabular}{l|} 
S \\
No
\end{tabular} & Title & Author & Technique Used & $\begin{array}{l}\text { Evaluation } \\
\text { Metrics (\%) }\end{array}$ & Remarks \\
\hline 1 & $\begin{array}{l}\text { Handwritten Dig- } \\
\text { its Recognition } \\
\text { Using Machine } \\
\text { learning }\end{array}$ & \begin{tabular}{l}
\multicolumn{2}{l}{ ShikharTandon, } \\
Shadab Akhter, \\
VaibhavPratap \\
Singh Nizam \\
Uddin $\quad$ Khan, \\
2019
\end{tabular} & $\begin{array}{l}\text { Describes the } \\
\text { recognition of } \\
\text { handwritten } \\
\text { scanned digits } \\
\text { using Machine } \\
\text { Learning meth- } \\
\text { ods with the help } \\
\text { of TensorFlow. }\end{array}$ & $\begin{array}{l}\text { Training:98 } \\
\text { Testing: } 97\end{array}$ & - \\
\hline 2 & $\begin{array}{l}\text { A Literature } \\
\text { Survey on Hand- } \\
\text { written Character } \\
\text { Recognition }\end{array}$ & $\begin{array}{l}\text { AyushPurohit , } \\
\text { Shardul Singh } \\
\text { Chauhan,2016 }\end{array}$ & $\begin{array}{l}\text { Provides a com- } \\
\text { parision on how } \\
\text { recognition is } \\
\text { used on different } \\
\text { characters based } \\
\text { on language } \\
\text { with different } \\
\text { techniques }\end{array}$ & $\begin{array}{l}\text { Accuracy: } \\
98.4\end{array}$ & $\begin{array}{l}\text { This paper does } \\
\text { not provide con- } \\
\text { crete methods on } \\
\text { how the recogni- } \\
\text { tion is performed } \\
\text { but provides an } \\
\text { overview on dif- } \\
\text { ferent techniques } \\
\text { that can be used }\end{array}$ \\
\hline 3 & \begin{tabular}{l}
\multicolumn{3}{l}{ Handwritten } \\
Digit Recog- \\
nition using \\
Machine and \\
Deep Learning \\
Algorithms
\end{tabular} & $\begin{array}{l}\text { Ritik Dixit, } \\
\text { RishikaKushwah, } \\
\text { SamayPashine, } \\
2020\end{array}$ & $\begin{array}{l}\text { Provides differ- } \\
\text { ent algorithms } \\
\text { that are used to } \\
\text { perform digit } \\
\text { recognition and } \\
\text { compares their } \\
\text { accuracies }\end{array}$ & $\begin{array}{l}\text { SVM } \\
\text { Training:99.98 } \\
\text { Testing } 94.05 \\
\text { MLP } \\
\text { Training:99.92 } \\
\text { Testing } 98.85 \\
\text { CNN } \\
\text { Training:99.53 } \\
\text { Testing } 99.31\end{array}$ & - \\
\hline 4 & $\begin{array}{l}\text { Handwritten } \\
\text { Character Recog- } \\
\text { nition using Neu- } \\
\text { ral Network and } \\
\text { Tensor Flow }\end{array}$ & $\begin{array}{l}\text { Megha Agarwal, } \\
\text { Shalika, Vinam- } \\
\text { Tomar, Priyanka } \\
\text { Gupta,2019 }\end{array}$ & $\begin{array}{l}\text { It clearly states } \\
\text { how CNN and } \\
\text { Tensor can iden- } \\
\text { tify different of- } \\
\text { fline handwritten } \\
\text { character. }\end{array}$ & $\begin{array}{l}\text { Training:94 } \\
\text { Testing: } 90.5\end{array}$ & $\begin{array}{l}\text { Performs digit } \\
\text { recognition but } \\
\text { accuracy is not } \\
\text { very high }\end{array}$ \\
\hline
\end{tabular}




\begin{tabular}{|c|c|c|c|c|c|}
\hline 5 & $\begin{array}{l}\text { Improved Hand- } \\
\text { written Digit } \\
\text { Recognition } \\
\text { Using Convo- } \\
\text { lutional Neural } \\
\text { Networks (CNN) }\end{array}$ & $\begin{array}{l}\text { SavitaAhlawat, } \\
\text { Amit Choudhary, } \\
\text { AnandNayyar, } \\
\text { Saurabh Singh } \\
\text { and Byungun } \\
\text { Yoon,2020 }\end{array}$ & $\begin{array}{l}\text { CNNs are very } \\
\text { effective in } \\
\text { perceiving the } \\
\text { structure of hand- } \\
\text { written charac- } \\
\text { ters/words in } \\
\text { ways that help } \\
\text { in automatic } \\
\text { extraction of } \\
\text { distinct features } \\
\text { and make CNN } \\
\text { the most suitable } \\
\text { approach for } \\
\text { solving handwrit- } \\
\text { ing recognition } \\
\text { problems. }\end{array}$ & $\begin{array}{l}\text { Training:99.87 } \\
\text { Testing: } 99.89\end{array}$ & $\begin{array}{l}\text { It is not very } \\
\text { time-efficient } \\
\text { and performance } \\
\text { of algorithm } \\
\text { decreases when } \\
\text { time used for } \\
\text { execution is } \\
\text { decreased }\end{array}$ \\
\hline 6 & $\begin{array}{l}\text { Handwritten } \\
\text { Optical Character } \\
\text { Recognition } \\
\text { (OCR): A Com- } \\
\text { prehensive Sys- } \\
\text { tematic Literature } \\
\text { Review (SLR) }\end{array}$ & $\begin{array}{l}\text { JamshedMemon, } \\
\text { Maira Sami, } \\
\text { Rizwan Ahmed- } \\
\text { Khan,Mueen } \\
\text { Uddin, } 2020\end{array}$ & $\begin{array}{l}\text { Provides analysis } \\
\text { based on articles } \\
\text { and the collected } \\
\text { data is analysed } \\
\text { and synthesized. }\end{array}$ & $\begin{array}{l}\text { Accuracy: } \\
99.6\end{array}$ & $\begin{array}{l}\text { Provides an } \\
\text { overview of } \\
\text { optical character } \\
\text { recognition but } \\
\text { does not provide } \\
\text { solution for the } \\
\text { problems }\end{array}$ \\
\hline 6 & $\begin{array}{l}\text { Handwritten } \\
\text { Optical Character } \\
\text { Recognition } \\
\text { (OCR): A Com- } \\
\text { prehensive Sys- } \\
\text { tematic Literature } \\
\text { Review (SLR) }\end{array}$ & $\begin{array}{l}\text { JamshedMemon, } \\
\text { Maira Sami, } \\
\text { Rizwan Ahmed- } \\
\text { Khan,Mueen } \\
\text { Uddin, } 2020\end{array}$ & $\begin{array}{l}\text { Provides analysis } \\
\text { based on articles } \\
\text { and the collected } \\
\text { data is analysed } \\
\text { and synthesized. }\end{array}$ & $\begin{array}{l}\text { Accuracy: } \\
99.6\end{array}$ & $\begin{array}{l}\text { Provides an } \\
\text { overview of } \\
\text { optical character } \\
\text { recognition but } \\
\text { does not provide } \\
\text { solution for the } \\
\text { problems }\end{array}$ \\
\hline
\end{tabular}

Then, the components of the picture and calculation in the network are decreased by utilizing the pooling layer. Besides, MAX-pooling is utilized in which just the most extreme worth from a pool is kept. Besides, in this layer, the profundity of the network stays unaltered. The pool-size $(2,2)$ is kept with a step of 2 , so every 4 pixels will turn into a solitary pixel. To stay away from overfitting in the model, the Dropout layer is utilized which drops a few neurons which are picked randomly so the model can be streamlined. The likelihood of a hub getting exited is set to 0.25 or $25 \%$. Following it, Flatten Layer is utilized which includes producing a segment grid (vector) from the 2dimensional framework. This section vector will be taken care of into the completely associated layer [15]. This layer comprises of 128 neurons with a dropout likelihood of 0.5 or half. Subsequent to applying the ReLu activation work, the yield is taken care of into the last layer of the model.

\section{Conclusion}

This paper differentiates their exactness and execution time and the help of exploratory outlines for a perspicuous course of action. In the wake of executing all of the models, it has been found that SVM has the most raised precision on getting ready information while on testing dataset $\mathrm{CNN}$ accomplishes the most limited exactness. Moreover, it has contrasted the execution time with acquiring understanding into the working of the algo- 
rithms. The SVM gave a proficient preparing exactness with a testing precision of over $90 \%$. In any case, to simply ensure that the model is impeccable, this paper rearranges the dataset by utilizing a train test split and it actually gives the precision over $90 \%$ making the model great. Therefore this paper incorporates one of the correctnesses given by the SVM. The future improvement of uses dependent on algorithms of deep and machine learning is basically vast. Later on, work on a denser or half and half algorithm than the current arrangement of algorithms with more complex information to accomplish the answers for some issues should be possible [17]. Later on, the utilization of these algorithms lies from the general population to undeniable level authorities. These algorithms can be utilized in clinics application for clinical treatment and monitoring the patients. The progression in this field can assist with establishing a environment of wellbeing, mindfulness, and comfort by utilizing these algorithms in the everyday application.

\section{References}

[1] Agarwal M, Shalika VT, Gupta P. Handwritten character recognition using neural network and tensor flow. International Journal of Innovative Technology and Exploring Engineering (IJITEE). 2019 Apr;8(6S4):1445-8.

[2] Kumar G, Bhatia PK, Banger I. Analytical review of preprocessing techniques for offline handwritten character recognition. International Journal of Advances in Engineering Sciences. 2013 Jul;3(3):14-22.

[3] Espana-Boquera S, Castro-Bleda MJ, Gorbe-Moya J, Zamora-Martinez F. Improving offline handwritten text recognition with hybrid HMM/ANN models. IEEE transactions on pattern analysis and machine intelligence. 2010 Aug 19;33(4):767-79.

[4] Pal U, Sharma N, Wakabayashi T, Kimura F. Handwritten numeral recognition of six popular Indian scripts. InNinth International Conference on Document Analysis and Recognition (ICDAR 2007) 2007 Sep 23 (Vol. 2, pp. 749-753). IEEE.

[5] Bajaj R, Dey L, Chaudhury S. Devnagari numeral recognition by combining decision of multiple connectionist classifiers. Sadhana. 2002 Feb;27(1):59-72.

[6] Arora S, Bhattacharjee D, Nasipuri M, Basu DK, Kundu M. Combining multiple feature extraction techniques for handwritten Devnagari character recognition. In2008 IEEE Region 10 and the Third International Conference on Industrial and Information Systems 2008 Dec 8 (pp. 1-6). IEEE.

[7] Arica N, Yarman-Vural FT. Optical character recognition for cursive handwriting. IEEE transactions on pattern analysis and machine intelligence. 2002 Aug 7;24(6):801-13.

[8] Aparna KH, Subramanian V, Kasirajan M, Prakash GV, Chakravarthy VS, Madhvanath S. Online handwriting recognition for Tamil. InNinth international workshop on frontiers in handwriting recognition 2004 Oct 26 (pp. 438-443). IEEE.

[9] Purohit A, Chauhan SS. A literature survey on handwritten character recognition. IJCSIT) International Journal of Computer Science and Information Technologies. 2016;7(1):1-5.

[10] Shamim SM, Miah MB, Angona Sarker MR, Al Jobair A. Handwritten digit recognition using machine learning algorithms. Global Journal Of Computer Science And Technology. 2018 Apr 13;18(1):17-23.

[11] Ali I, Ali I, Subhash AK, Raza SA, Hassan B, Bhatti P. Sindhi Handwritten-Digits Recognition Using Machine Learning Techniques. Int. J. Comput. Sci. Netw. Secur. 2019 May;19:195-202.

[12] Ahlawat S, Choudhary A, Nayyar A, Singh S, Yoon B. Improved handwritten digit recognition using convolutional neural networks (CNN). Sensors. 2020 Jan;20(12):3344-50.

[13] O'Shea K, Nash R. An introduction to convolutional neural networks. arXiv preprint arXiv:1511.08458. 2015 Nov 26:274-82.

[14] Nwankpa C, Ijomah W, Gachagan A, Marshall S. Activation functions: Comparison of trends in practice and research for deep learning. arXiv preprint arXiv:1811.03378. 2018 Nov 8:422-8.

[15] Udofia U. Basic overview of convolutional neural network (cnn). Retrieved May. 2018;27: 578-85.

[16] Brownlee J. How do convolutional layers work in deep learning neural networks?. Machine Learning Mastery. 2020 Feb:313-9.

[17] Memon J, Sami M, Khan RA, Uddin M. Handwritten optical character recognition (OCR): A comprehensive systematic literature review (SLR). IEEE Access. 2020 Jul 28;8:142642-68. 